\title{
The Serological Diagnosis of Canine Leishmaniasis: Commercial ELISA or in House IFAT?
}

\author{
Pasquale Santoro', Andrea Vellusi ${ }^{2}$ \\ ${ }^{1}$ Università degli Studi di Napoli Federico II - Dipartimento di Pediatria, Napoli, Italy \\ ${ }^{2}$ Diagnostica di Laboratorio (Di.Lab.) S.r.I., Napoli, Italy \\ Email: pasantor@unina.it, andreavellusi@libero.it
}

Received 27 January 2015; accepted 12 February 2015; published 13 February 2015

Copyright (C 2015 by authors and Scientific Research Publishing Inc.

This work is licensed under the Creative Commons Attribution International License (CC BY). http://creativecommons.org/licenses/by/4.0/

(c) (i) Open Access

\begin{abstract}
The levels of anti-Leishmania infantum antibodies are relevant with the diagnosis and the followup of infected dogs. Both ELISA (Enzyme-Linked Immunosorbent Assay) and IFAT (Indirect Immuno-Fluorescent Antibody Test) may be employed to estimate the antibody levels in dogs, but at present, the correlation between ELISA absorbance and IFAT titer has not been investigated. In this paper, we compared the optical densities obtained with a commercial ELISA, Leiscan ${ }^{\circledR}$ (Laboratorios Dr. Esteve S.A.), versus the titers obtained by in house IFAT. We measured ELISA absorbance of: 44 IFAT negative samples coming from an endemic area; 10 negative samples coming from a non endemic area; 29 samples with an IFAT titer between $1 / 40$ and 1/80; 10 samples with an IFAT titer of 1/160; 9 samples with an IFAT titer of 1/320; 10 samples with an IFAT titer of $1 / 640 ; 10$ samples with an IFAT titer $\geq$ of $1 / 1280$. Results show that: a) there is a poor correlation between IFAT titer and ELISA absorbance; b) Leiscan ${ }^{\circledR}$ is not able to distinguish between IFAT negative samples and IFAT titers up to 1:160; c) IFAT negative samples from an endemic or non endemic area show different ELISA absorbance; d) the performance of the kit may be improved by the use of a more appropriate cut-off.
\end{abstract}

Keywords

ELISA, IFAT, Leishmania infantum

\section{Introduction}

Identification of infected dogs is crucial for the control of human leishmaniasis, as dogs act as the main reservoir

How to cite this paper: Santoro, P. and Vellusi, A. (2015) The Serological Diagnosis of Canine Leishmaniasis: Commercial ELISA or in House IFAT? Open Journal of Veterinary Medicine, 5, 30-34. http://dx.doi.org/10.4236/ojvm.2015.52005 
for the disease. Canine leishmaniasis is diagnosed directly through the presence of the parasite by direct microscopic examination of lymph nodes, bone marrow aspirates and/or culture of skin lesions, or indirectly by serological techniques. However, direct techniques lack of sensitivity, and interpretation of indirect serological techniques is often difficult, as they are more sensitive but less specific [1] [2]. Although PCR has been described as a method sensitive enough to detect all infected individuals, results may vary according to the sampled tissue. In addition, PCR appears too sensitive for mass screening and shows a low predictive value in detecting disease [3] [4]. Therefore, none of these methods is capable to give an unequivocal classification of the infected subject. A combination of serological and molecular methods may provide more reliable data.

Indirect Immune-Fluorescent Antibody Test (IFAT) is one of the most frequently used serological methods, and it is considered by some authors as the "gold standard" [5] [6]. However, IFAT is time consuming and has been described as an operator-dependent method. For this reason, a number of Enzyme-Linked Immunosorbent Assay (ELISA) approaches have been developed. Commercial ELISA may differ in antigen extraction and preparation, may use recombinant antigens or may be different in classes of polyclonal anti-dog immunoglobulin IgG. Each method has some disadvantages [7].

Since IFAT has been employed for years for the detection of anti-Leishmania antibodies, commercial ELISA frequently provides a table of correspondence between results obtained with ELISA and IFAT. In this report, we compared the optical density (O.D.) obtained with a commercial ELISA with the antibody levels obtained by IFAT. We employed a recent commercialized ELISA kit, Leiscan ${ }^{\circledR}$ (Laboratorios Dr. Esteve S.A.), which was described as the best commercialized ELISA kit, and considered by some authors as "the new gold standard" of the serological techniques [8]. To our best knowledge, this is the first paper that compares ELISA O.D. with IFAT titers, clearly demonstrating that results obtained with ELISA and IFAT are not comparable. Moreover, in this paper we show evidence that the major limitation of "commercial" ELISA is related to the choice of an appropriate cut-off.

\section{Materials and Methods}

\subsection{ELISA}

ELISA was performed as described by the manufacturer. All steps were performed at $20^{\circ} \mathrm{C}-25^{\circ} \mathrm{C}$ and under continuous gentle agitation. Briefly: $100 \mu \mathrm{l}$ of diluted samples (1:20), controls (negative, high positive and low positive), were added to the appropriate well and incubated 10 minutes. Wells were washed five times with 300 $\mu \mathrm{l}$ of washing solution and $100 \mu \mathrm{l}$ of conjugate was added to each well. After 5 minutes, the wells were washed again and $100 \mu \mathrm{l}$ of substrate were added. The reaction was stopped after 10 minutes by adding a stopping solution. Microplates were read by a microplate spectrophotometer (EPOCH, BioTek U.S. Winooski, VT 05405, USA). Results were expressed confronting the O.D. of the sample with the O.D. of the cut off included in the kit. No justification or supporting evidence was provided for the selection of such cut-off.

\subsection{IFAT}

IFAT was performed employing "in house prepared antigen" obtained by culture of promastigotes of Leishmania infantum obtained from a dog that acquired the infection naturally and had not received treatment. The assay procedure followed the protocol of the Office International des Epizooties [9]. Anti-Leishmania antibodies were detected using rabbit anti-dog IgG conjugated to fluorescein isothiocyanate (Sigma Chemical; St. Louis, MO, U.S.A.). Each sample was run in duplicate and was read by two persons with specific experience in the field. Observation was performed at a magnification of $400-600 x$.

The control sera included in the ELISA kit was also examined by IFAT.

\subsection{Recruitment of Samples}

IFAT negative samples were collected from clinically healthy dogs showing normal blood count and normal serum levels of urea, creatinine, AST, ALT, and total proteins. The samples also showed a normal ratio of both serum albumin/globulin and proteinuria/creatinuria and resulted serologically negative for Ehrlichia canis and Rickettsia rickettsii. The sera labelled "endemic area" were from Napoli (Campania, southern region of Italy), whereas those labelled "non endemic area" where from Padova (Veneto, northern region of Italy).

Leishmania infantum IFAT positive sera were selected from the samples routinely analyzed in our specialized 
laboratory of veterinary analysis, and coming from the endemic area of Napoli.

Results were reported as mean $(\overline{\mathrm{x}}) \pm$ standard deviation (S.D.). The data were analyzed with the help of a commercial software (GraphPad PRISM; GraphPad Software Incorporated, San Diego, CA). The one-way analysis of variance, together with the Tukey post hoc test was used to compare different groups of data. A p $<0.01$ was considered statistically significant.

\section{Results and Discussion}

Table 1 shows the ELISA absorbance at $\lambda=450 \mathrm{~nm}$ of cut-off and sera.

As shown, the O.D. of the cut-off included in the kit is $0.560 \pm 0.163$, with an IFAT titer of 1:320. This value is approximately two-fold higher than the $\bar{x}$ O.D. of IFAT samples with a titer of 1:80. There is a wide agreement to consider IFAT titers $\geq 1: 160$ as "positive", and titers $\leq 1: 80$ as "suspicious" [1] [2] [4] [10]. Hence, by using the standard included in the kit Leiscan ${ }^{\circledR}$, a lot of IFAT positive/suspicious samples will be considered as negative one (Table 1; Sample ratio 1).

It has been reported [2] [11] that an appropriate ELISA cut-off may be the O.D. ( $\bar{x}+2$ S.D.) of a pool of noninfected dogs (IFAT negative). It has also been reported [1] that the choice of an appropriate cut-off as well the epidemiological characteristics of the area under study may influence the sensitivity and the specificity of a method. Accordingly, we found different ELISA absorbance in negative samples coming from different geographical areas (e.g. endemic or non-endemic). This data strongly suggests that the ELISA cut-off should be related to the area of provenience of the samples. The choice of the correct cut-off is a pivotal step for the performance of ELISA; and a wrong choice will results in a misinterpretation of results. From this point of view, the cut-off included in the kit is inadequate. This fact is well depicted in Table 1. As shown, IFAT positive samples up to a titer of 1:160 will results as negative one by Leiscan ${ }^{\circledR}$ by using the cut-off included in the kit (sample ratio 1). However, the use of a cut-off $(\bar{x}+2$ S.D; O.D.) related to a pool of sera coming from IFAT negative healthy dogs (endemic area; Sample ratio 2, or non-endemic area, Sample ratio 3) dramatically increases the significance of the sample ratio. Using this criterion, IFAT titers of 1:80 and 1:160 appeared as "suspicious" or as "positive" by ELISA (Table 1; Sample ratio 2-3), accordingly with the cut-off considered (endemic or non-endemic). Differently from ELISA, an advantage of an end-point IFAT titration is that the clinician, not the laboratory, may assign the appropriate weight to the obtained results, accordingly with the followup and the geographical area of provenience of the samples.

Table 1. Correlation between ELISA absorbances and IFAT titers. Sorting was based on IFAT titers. Results were reported as mean \pm S.D. Sample ratio (1) was obtained dividing the sample O.D. with the O.D. of the cut-off included in the ELISA kit $(\overline{\mathbf{X}})$. Sample ratio (2) was obtained using as cut-off the pool of negative samples from an endemic area $(\overline{\mathbf{X}}+2$ S.D.). Sample ratio (3) was obtained using as cut-off the pool of negative samples from a non-endemic area ( $\bar{X}+2$ S.D). As reported in the procedure of "Leiscan ${ }^{\circledR}$ ", a value between 0.9 and 1.1 should be considered as "suspicious"; and a value between 1.1 and 1.5 should be considered as a "low positive". The column ANOVA reports the "p" value of the O.D. of the considered group in comparison with group 1 (negative samples from endemic area).

\begin{tabular}{|c|c|c|c|c|c|c|}
\hline Group & IFAT Titer & $\begin{array}{l}\text { ELISA O.D. } \\
(\lambda=450 \mathrm{~nm})\end{array}$ & ANOVA & $\begin{array}{l}\text { Sample } \\
\text { ratio (1) }\end{array}$ & $\begin{array}{l}\text { Sample } \\
\text { ratio (2) }\end{array}$ & $\begin{array}{l}\text { Sample } \\
\text { ratio (3) }\end{array}$ \\
\hline 1 & Negative (endemic area; $n=44$ ) & $0.127 \pm 0.07$ & & & & \\
\hline 2 & Negative (non-endemic area; $\mathrm{n}=10$ ) & $0.092 \pm 0.06$ & & & & \\
\hline 3 & $1: 40(\mathrm{n}=12)$ & $0.168 \pm 0.105$ & $\mathrm{p}>0.05$ & 0.30 & 0.62 & 0,79 \\
\hline 4 & $1: 80(\mathrm{n}=17)$ & $0.247 \pm 0.175$ & $\mathrm{p}>0.05$ & 0.44 & 0.92 & 1.16 \\
\hline 5 & $1: 160(\mathrm{n}=10)$ & $0.306 \pm 0.177$ & $\mathrm{p}>0.05$ & 0.54 & 1.14 & 1.44 \\
\hline 6 & $1: 320(\mathrm{n}=9)$ & $1.28 \pm 0.52$ & $\mathrm{p}<0.01$ & 2.28 & 4.79 & 6.03 \\
\hline 7 & $1: 640(\mathrm{n}=10)$ & $2.27 \pm 0.72$ & $\mathrm{p}<0.01$ & 4.05 & 8.5 & 10.7 \\
\hline 8 & $\geq 1280(\mathrm{n}=10)$ & Out of range & --- & --- & --- & --- \\
\hline Cut-off & $1: 320(\mathrm{n}=3)$ & $0.560 \pm 0.163$ & & & & \\
\hline
\end{tabular}


Statistical analysis also shows that ELISA is not able to differentiate IFAT titers between 1:40 and 1:160 (ANOVA; $p$ > 0.05). This is due to the wide S.D. of ELISA O.D., as shown in Table 1. We have also to consider that IFAT allows distinguishing between different patterns of fluorescence, which not necessarily may generate similar ELISA absorbance. Taken together, these facts suggest that ELISA and IFAT are methods that may not necessarily generate comparable results. Finally, Table 1 shows that ELISA requires further dilution steps to measure samples with a high amount of anti-leishmania antibodies (IFAT titers $\geq 1: 1280$ ). From this point of view, the quantitation of high antibody levels needs a further dilution step by using either ELISA or IFAT, but results will remain not comparable.

\section{Conclusions}

In conclusion, this paper show evidence that Leiscan ${ }^{\circledR}$ is not suitable for the detection of anti-leishmania antibodies in dog serum mainly due to the inappropriate choice of the cut-off included in the kit. The availability of an appropriate cut-off represents an intrinsic limit for each laboratory that wants to perform the quantitation of anti-leishmania antibodies by a "commercial" ELISA. Other authors have investigated the best way to determine the cut-off value for serological assays like arbitrary methods, methods to optimize sensitivity or specificity, methods to optimize test accuracy, ROC curves, and methods to optimize the predictive value. The discussion about which of these methods is the best one for the determination of anti-leishmania antibodies is far from the aim of this paper.

However, our results suggest that the performance of the kit may be improved by using a cut-off related to a pool of sera collected from non infected dogs. Moreover, the cut-off should be related to the geographical area of provenience of samples. Our results also show that a commercial ELISA does not offer any particular advantage in the quantitation of anti-Leishmania antibodies, especially with low positive sera, as it is not able to differentiate titers ranging between 1:40 and 1:160 (Table 1, ANOVA, $p>0.05$ ).

It is our opinion that, at the present time, there are no reasons to replace an appropriately well-performed in house IFAT with a "commercial" ELISA.

Finally, we have to consider that it is reasonable to suppose that the pool of IFAT-negative healthy dogs used as cut-off is from non-infected subjects. With stronger reasons, this assumption is correct for dogs coming from a non-endemic area. However, we have no certainty that IFAT negative samples belong to non-infected dogs, as we do not perform PCR for the detection of Leishmania spp. This fact does not invalidate our conclusions, because this report is focused on the ability of each test to reveal the presence of antibody levels in the sera and on the existence of a correlation between IFAT titers and ELISA absorbances, rather than on the ability of these methods to detect infected dogs.

\section{Acknowledgements}

The authors greatly acknowledge Michela Pasquinucci and Emiliana Cigliano for their skilled technical assistance.

\section{Conflict of Interest}

None of the authors has a financial or personal relationship with other people or organizations that could inappropriately influence or bias the content of the paper.

\section{Funding}

The authors received no financial support for the research, authorship, and/or publication of this article.

\section{References}

[1] Morales-Yuste, M., Morillas-Márquez, F., Díaz-Sáez, V., Barón-López, S., Acedo-Sánchez, C. and Martín-Sánchez, J. (2012) Epidemiological Implications of the Use of Various Methods for the Diagnosis of Canine Leishmaniasis in Dogs with Different Characteristics and in Differing Prevalence Scenarios. Parasitology Research, 111, 155-164. http://dx.doi.org/10.1007/s00436-011-2812-7

[2] Rodríguez-Cortés, A., Ojeda, A., Francino, O., López-Fuertes, L., Timón, M. and Alberola, J. (2010) Leishmania Infection: Laboratory Diagnosing in the Absence of a "Gold Standard". The American Journal of Tropical Medicine and 
Hygiene, 82, 251-256.

[3] Lachaud, L., Chabbert, E., Dubessay, P., Dereure, J., Lamothe, J., Dedet, J.P. and Bastien, P. (2002) Value of Two PCR Methods for the Diagnosis of Canine Visceral Leishmaniasis and the Detection of Asymptomatic Carriers. Parasitology, 125, 197-207. http://dx.doi.org/10.1017/S0031182002002081

[4] Ashford, D.A., Bozza, M., Freire, M., Mirando, J.C., Sherlock, I., Eulalio, C., Lopes, U., Fernande, O., Degrave, W., Baker, R.H., Badaro, R. and David, J.R. (1995) Comparison of the Polymerase Chain Reaction and Serology for the Detection of Canine Visceral Leishmaniasis. The American Journal of Tropical Medicine and Hygiene, 53, $251-255$.

[5] Reis, A.B., Martins-Filho, O.A., Teixeira-Carvalho, A., et al. (2009) Systemic and Compartmentalized Immune Response in Canine Visceral Leishmaniasis. Veterinary Immunology and Immunopathology, 128, 87-95. http://dx.doi.org/10.1016/j.vetimm.2008.10.307

[6] Maia, C. and Campino, L. (2009) Methods for Diagnosis of Canine Leishmaniasis and Immune Response to Infection.. Veterinary Parasitology, 158, 274-287. http://dx.doi.org/10.1016/j.vetpar.2008.07.028

[7] Rosati, S., Ortoffi, M., Profiti, M., Mannelli, A., Mignone, W., Bollo, E. and Gradoni, L. (2003) Prokaryotic Expression and Antigenic Characterization of Three Recombinant Leishmania Antigens for Serological Diagnosis of Canine Leishmaniasis. Clinical and Vaccine Immunology, 10, 1153-1156.

[8] Rodríguez-Cortés, A., Ojeda, A., Todolí, F. and Alberola, J. (2013) Performance of Commercially Available Serological Diagnostic Tests to Detect Leishmania infantum Infection on Experimentally Infected Dogs. Veterinary Parasitology, 191, 363-366. http://dx.doi.org/10.1016/j.vetpar.2012.09.009

[9] World Organisation for Animal Health (OIE): Leishmaniosis (2008) Manual of Diagnostic Tests and Vaccines for Terrestrial Animals. 6th edition, 241-243.

[10] Proverbio, D., Spada, E., Baggiani, L., De Giorgi, G.B. and Perego, R. (2013) Comparison of a Clinic-Based ELISA Test Kit with the Immunofluorescence Antibody Test for Assaying Leishmania infantum Antibodies in Dogs. BioMed Research International, 2013, Article ID: 249010. http://dx.doi.org/10.1155/2013/249010.

[11] Barajas-Rojas, J.A., Riemann, H.P. and Franti, C.E. (1993) Notes about Determining the Cut-Off Value in Enzyme Linked Immunosorbent Assay (ELISA). Preventive Veterinary Medicine, 15, 231-233.

http://dx.doi.org/10.1016/0167-5877(93)90116-B 